\title{
THREE POSSIBLE PLANETARY NEBULAE FROM NEAR-IR OBSERVATIONS
}

\author{
P. PERSI, A. MARENZI, A. PREITE-MARTINEZ and M. FERRARI-TONIOLO \\ Istituto di Astrofisica Spaziale, CP 67, 00044 Frascati, Italy
}

A catalogue of 388 new possible planetary nebulae was selected by Preite-Martinez (1988) from the IRAS Point Source Catalogue. These unidentified sources have IRAS colours in the range $F(12) / F(25) \leq 0.35$ and $F(25) / F(60) \geq 0.35$, and are located in the proximity of the galactic equator $\left(|b| \leq 15^{\circ}\right)$. In order to identify these IRAS sources we have undertaken a programme of near-IR observations using an InSb photometer and a nearIR camera. We report here on results relative to four of these sources.

A scanning at $2.2 \mu$ within the positional error box of IRAS sources 12262-6417, 12302-6317, and 12316-6401 was made using the InSb photometer at the ESO $2.2 \mathrm{~m}$ telescope, with an aperture of 8 arcsec. Photometry in the J, H, K, L, and M filters was obtained for each source.

The J-H, H-K colours of IRAS $12262-6417$ and $12302-6317$ show a strong IR excess, interpreted in terms of dust emission at a temperature of $1000-1500 \mathrm{~K}$. Only a background star was found in the field of IRAS 12316-6401. The IR energy distributions of IRAS 12262-6417 and 12302-6317 both show a hot as well as a cold component. A similar energy distribution has been observed in symbiotic stars, and in He 2-104 by Schwarz et al. (1989).

$\mathrm{J}, \mathrm{H}$, and $\mathrm{K}$ broad-band images of IRAS $11339-6004$ were collected with the ESO $\mathrm{Hg}: \mathrm{Cd}$ :Te $64 \times 64$ pixels array camera (IRAC- 1 ) at the same telescope. The images were obtained with a scale of $0.8 \mathrm{arcsec} / \mathrm{pix}$ in beam-switching mode, and were calibrated using standard stars. Using IRAF packages we have derived $\mathrm{J}, \mathrm{H}, \mathrm{K}$ photometry of the four sources closer to the IRAS position. Only one of these show near-IR colours typical of an N-type planetary nebula (Whitelock, 1985; Persi et al, 1987).

The source that we associate with IRAS 11339-6004 shows an energy distribution dominated by nebular emission in the near-IR and by a cold component at $\mathrm{T}_{C}=228 \mathrm{~K}$.

\section{References}

Persi, P., Preite-Martinez, A., Ferrari-Toniolo, M., Spinoglio, L. 1987, Planetary and protoplanetary nebulae: from IRAS to ISO, ed. A. Preite-Martinez, Reidel Pub.Co., p.221 Preite-Martinez, A. 1989, Astron. Astrophys. Suppl. Ser. 76, 317

Schwarz, H.E., Aspin, C., Lutz, J.H. 1989, Astrophys. J. Lett. 344, L29

Whitelock, P.A. 1985, Mon. Not. Royal Astron. Soc. 213, 59 